\title{
Is spontaneous coronary artery dissection (SCAD) related to vascular inflammation and epicardial fat? -insights from computed tomography coronary angiography
}

\author{
Jeremy Yuvaraj ${ }^{1}$, Andrew Lin ${ }^{1}$, Nitesh Nerlekar ${ }^{1}$, Hashrul Rashid ${ }^{1}$, James D. Cameron ${ }^{1}$, Sujith Seneviratne ${ }^{1}$, \\ Stephen Nicholls ${ }^{1,2}$, Peter J. Psaltis ${ }^{1,2}$, Dennis T. L. Wong ${ }^{1,2}$ \\ ${ }^{1}$ Monash Cardiovascular Research Centre, Monash University and MonashHeart, Monash Health, Clayton, Victoria, Australia; ${ }^{2}$ South Australian \\ Health and Medical Research Institute, Adelaide, Australia \\ Correspondence to: Associate Professor Dennis T. L. Wong, MBBS, MD, PhD. Monash Cardiovascular Research Centre, MonashHeart, Monash \\ Health, Victoria, Australia. Email: dennis.wong@monash.edu.
}

Submitted Aug 28, 2019. Accepted for publication Dec 09, 2019.

doi: $10.21037 /$ cdt.2020.01.09

View this article at: http://dx.doi.org/10.21037/cdt.2020.01.09

Spontaneous coronary artery dissection (SCAD) is a rare coronary condition characterized by a non-traumatic, nonatherosclerotic separation of the layers of the arterial wall and the formation of a false lumen. The role of vascular inflammation in the pathophysiology of the disease remains unclear. Inflammation within the coronary vasculature results in the release of inflammatory mediators that affect the surrounding adipose tissue (1) and has been linked to atherosclerosis. Epicardial adipose tissue (EAT) is best defined as the fatty tissue enclosed within the pericardial sac-between the epicardial surface of the heart and the visceral pericardium (2). Pericoronary adipose tissue (PCAT) is a subset of EAT that directly encases the coronary arteries. These adipose tissue depots can be investigated using routine computed tomography coronary angiography (CTCA). On CTCA, EAT volume (EFV) has been positively associated with coronary artery disease (3), while increased PCAT attenuation has demonstrated significant prognostic value for cardiac mortality and is now a well-validated imaging biomarker of vascular inflammation (4). Our study investigated EFV, density and PCAT attenuation as CTCA markers of vascular inflammation in the context of SCAD.

SCAD patients and asymptomatic controls with no acute coronary syndrome who had undergone 320-detector CTCA within 2 weeks of acute presentation were studied. Both groups were evaluated for EFV, EAT density (EFD) and PCAT attenuation. For all analysis, adipose tissue was defined as voxels between -190 and -30 Hounsfield units $(\mathrm{HU})$ within a volume of interest (5). Semi-automated software was used to quantitatively measure EFV and EFD (QFAT version 2.0), and PCAT (AutoPlaque version 2.0). EFV was measured in milliliters $(\mathrm{mL})$ and EFD was measured in $\mathrm{HU}$, and these were quantified by contouring the visceral pericardium between an upper and lower border (bifurcation of the pulmonary trunk, and appearance of the liver, respectively). PCAT attenuation was also measured in $\mathrm{HU}$ and quantified around the proximal right coronary artery (RCA) (10-50 $\mathrm{mm}$ from the ostium), as validated previously (4). PCAT was analyzed radially outwards in three-dimensional layers from the outer vessel wall within a distance of $3 \mathrm{~mm}$, the typical diameter of the RCA. Normality was assessed via the Shapiro-Wilk test and differences in traditional cardiovascular risk factors between groups using the Fisher exact test. Median differences between groups were compared using the Mann-Whitney U test. Ethics approval for this study was obtained from the Monash Health Human Research Ethics Committee (HREC).

A total of 11 patients with confirmed SCAD (52.4 \pm

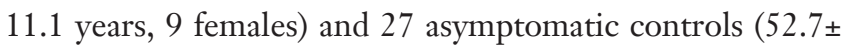
11.0 years, 7 females) were assessed. No significant difference was found between groups in cardiovascular risk factors such as diabetes mellitus $(\mathrm{P}=0.13)$, dyslipidemia $(\mathrm{P}=0.15)$, hypertension $(\mathrm{P}=0.46)$, smoking status $(\mathrm{P}=1.0)$ and family history of IHD $(\mathrm{P}=0.46)$. We found no significant difference between SCAD patients and controls in median EFV [(60.5 mL, interquartile range (IQR), 40.6 to $72.0 \mathrm{~mL}$; versus $54.5 \mathrm{~mL}$, IQR, 34.8 to $69.6 \mathrm{~mL} ; \mathrm{P}=0.49$ ] and median EFD (-85.7 HU, IQR, -86.95 to $-83.79 \mathrm{HU}$; versus -83.0 

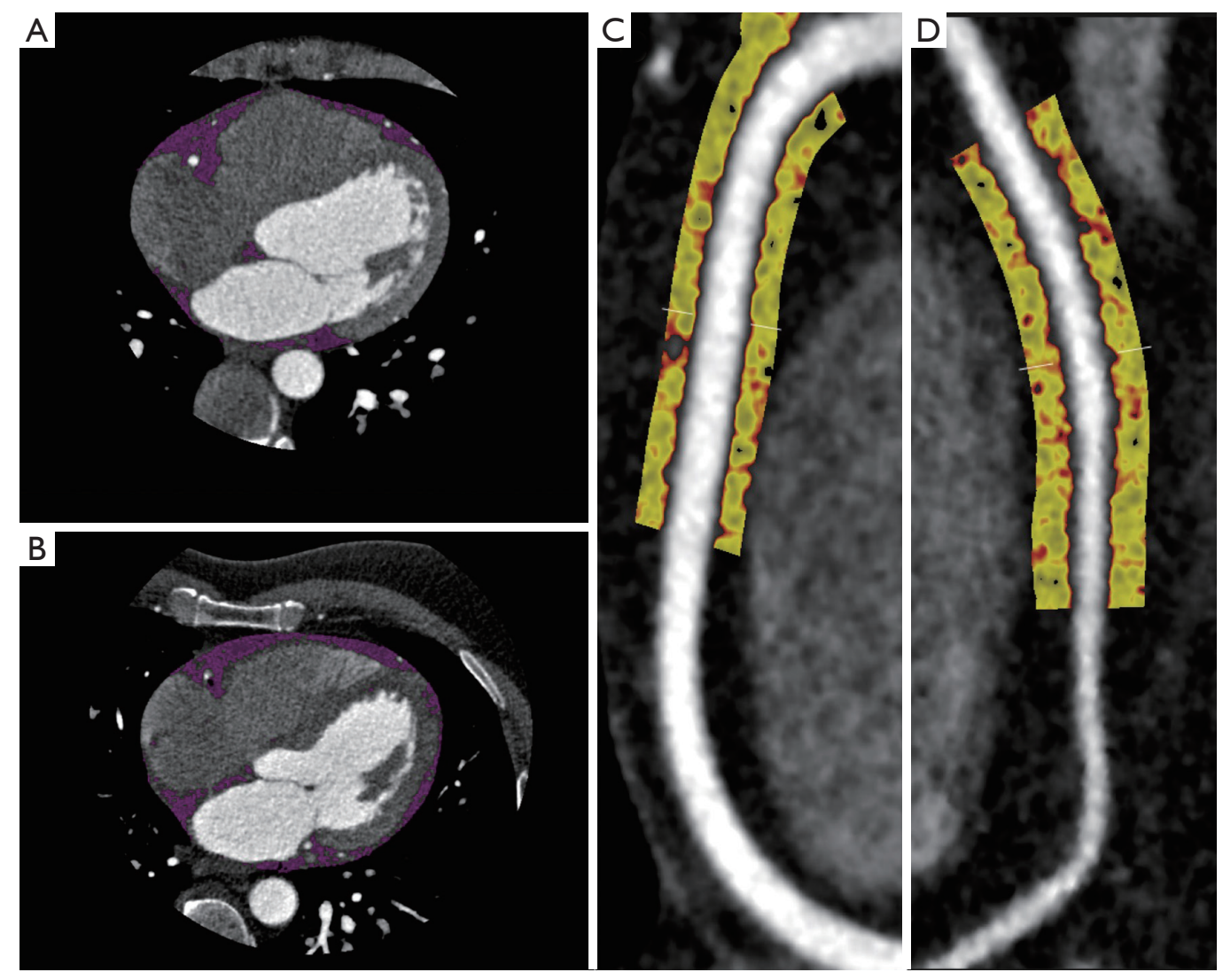

Figure 1 Comparison of EAT and PCAT in a SCAD patient versus asymptomatic control as shown on CTCA. EAT (purple) shown in cross-section of the heart in SCAD (A) versus control (B). PCAT shown in straightened view of the proximal RCA in SCAD (C) versus control (D), with colour map corresponding to CT attenuation in Hounsfield units (HU) ranging from -190 HU (yellow) to -30 HU (red). EAT, epicardial adipose tissue; PCAT, pericoronary adipose tissue; SCAD, spontaneous coronary artery dissection; CTCA, computed tomography coronary angiography; HU, Hounsfield units; RCA, right coronary artery.

HU, IQR, -87.5 to $-77.4 \mathrm{HU} ; \mathrm{P}=0.34)$. Likewise, median PCAT attenuation was not significantly different between SCAD patients (-91.7 HU, IQR, -94.9 to $-87.2 \mathrm{HU})$ and controls $(-88.4 \mathrm{HU}, \mathrm{IQR},-91.3$ to $-82.8 \mathrm{HU}$; $\mathrm{P}=0.1)$. A visual representation of EAT and PCAT in a SCAD and control patient is shown in Figure 1.

To our knowledge, this is the first paper investigating vascular inflammation specifically in a SCAD cohort. We report no significant difference between SCAD patients and asymptomatic controls in validated biomarkers of vascular inflammation using routine CTCA. Previous studies on this subject often consist of small case series, or provide limited evidence for vascular inflammation in the disease. One study reported two cases of SCAD with increased attenuation of epicardial fat as a result of local inflammatory processes (defined as perivascular "fat stranding") (6). Another study investigated 14 SCAD patients with CTCA performed within two days of angiographic diagnosis, but found increased epicardial fat attenuation in a total of six cases, including a case of multi-vessel SCAD in which increased attenuation was detected in only one of the affected segments (7). With inflammation being present in only a proportion of these SCAD cases, it is difficult to postulate what role, if any, inflammation plays in the disease. Highrisk atherosclerotic plaque has been associated with CT markers of inflammation in the form of epicardial fat (3) and PCAT (8). Unlike atherosclerosis, however, patients with SCAD often present without traditional risk factors for cardiovascular disease. Eosinophilic inflammatory infiltrates have been reported in histological examinations of SCAD cases (9), but a non-invasive, antemortem method of detecting vascular inflammation would be of significant value. Early detection of SCAD would enable effective planning of clinical intervention or medical therapy to be administered 
before the onset of a coronary event. However, there is a paucity of literature on inflammation detected via CTCA in SCAD, likely due to the rarity of the disease itself. The findings of our study indicate that vascular inflammation may harbour a limited role in the pathophysiology of SCAD, but larger studies are needed to validate these findings.

\section{Acknowledgments}

Funding: A Lin is supported by National Heart Foundation Postgraduate Scholarship; N Nerlekar is supported by National Heart Foundation Postdoctoral Fellowship; $\mathrm{H}$ Rashid is supported by National Heart Foundation Health Practitioner Fellowship; S Nicholls is supported by NHMRC Principal Research Fellowship; PJ Psaltis and DT Wong are supported by NHF Future Leaders Fellowship.

\section{Footnote}

Conflicts of Interest: All authors have completed the ICMJE uniform disclosure form (available at http://dx.doi. org/10.21037/cdt.2020.01.09). HR reports grants from National Heart Foundation, grants from Royal Australasian College of Physician, grants from National Health and Medical Research Council, during the conduct of the study. SN reports grants from AstraZeneca, Amgen, Anthera, Eli Lilly, Esperion, Novartis, Cerenis, The Medicines Company, Resverlogix, InfraReDx, Roche, Sanofi-Regeneron and LipoScience, personal fees from AstraZeneca, Akcea, Eli Lilly, Anthera, Omthera, Merck, Takeda, Resverlogix, Sanofi-Regeneron, CSL Behring, Esperion, Boehringer Ingelheim, outside the submitted work. PP reports personal fees from ESPERION Therapeutics, Bayer, Boehringer Ingelheim, Merck, Pfizer, Astra Zeneca, grants from ABBOTT Vascular, outside the submitted work. The other authors have no conflicts of interest to declare.

Ethical Statement: The authors are accountable for all aspects of the work in ensuring that questions related to the accuracy or integrity of any part of the work are appropriately investigated and resolved.

Open Access Statement: This is an Open Access article distributed in accordance with the Creative Commons Attribution-NonCommercial-NoDerivs 4.0 International License (CC BY-NC-ND 4.0), which permits the noncommercial replication and distribution of the article with the strict proviso that no changes or edits are made and the original work is properly cited (including links to both the formal publication through the relevant DOI and the license). See: https://creativecommons.org/licenses/by-nc-nd/4.0/.

\section{References}

1. Antonopoulos AS, Sanna F, Sabharwal N, et al. Detecting human coronary inflammation by imaging perivascular fat. Sci Transl Med 2017;9:eaal2658.

2. Talman AH, Psaltis PJ, Cameron JD, et al. Epicardial adipose tissue: far more than a fat depot. Cardiovasc Diagn Ther 2014;4:416-29.

3. Nerlekar N, Brown AJ, Muthalaly RG, et al. Association of Epicardial Adipose Tissue and High-Risk Plaque Characteristics: A Systematic Review and Meta-Analysis. J Am Heart Assoc 2017;6:e006379.

4. Oikonomou EK, Marwan M, Desai MY, et al. Non-invasive detection of coronary inflammation using computed tomography and prediction of residual cardiovascular risk (the CRISP CT study): a post-hoc analysis of prospective outcome data. Lancet 2018;392:929-39.

5. Nerlekar N, Baey YW, Brown AJ, et al. Poor Correlation, Reproducibility, and Agreement Between Volumetric Versus Linear Epicardial Adipose Tissue Measurement: A 3D Computed Tomography Versus 2D Echocardiography Comparison. JACC Cardiovasc Imaging 2018;11:1035-6.

6. Hedgire S, Baliyan V, Zucker EJ, et al. Perivascular Epicardial Fat Stranding at Coronary CT Angiography: A Marker of Acute Plaque Rupture and Spontaneous Coronary Artery Dissection. Radiology 2018;287:808-15.

7. Tweet MS, Akhtar NJ, Hayes SN, et al. Spontaneous coronary artery dissection: Acute findings on coronary computed tomography angiography. Eur Heart J Acute Cardiovasc Care 2019;8:467-75.

8. Goeller M, Achenbach S, Cadet S, et al. Pericoronary Adipose Tissue Computed Tomography Attenuation and High-Risk Plaque Characteristics in Acute Coronary Syndrome Compared With Stable Coronary Artery Disease. JAMA Cardiol 2018;3:858-63.

9. Pitliya A, Datta S, Kalayci A, et al. Eosinophilic inflammation in spontaneous coronary artery dissection: A potential therapeutic target? Med Hypotheses 2018;121:91-4.

Cite this article as: Yuvaraj J, Lin A, Nerlekar N, Rashid H, Cameron JD, Seneviratne S, Nicholls S, Psaltis PJ, Wong DT. Is spontaneous coronary artery dissection (SCAD) related to vascular inflammation and epicardial fat? -insights from computed tomography coronary angiography. Cardiovasc Diagn Ther 2020;10(2):239-241. doi: 10.21037/cdt.2020.01.09 